\title{
Clinical response to dabrafenib plus trametinib in a pediatric ganglioglioma with BRAF p.T599dup mutation
}

\author{
Katherine E. Miller, ${ }^{1}$ Kathleen M. Schieffer, ${ }^{1}$ Olivia Grischow, ${ }^{1}$ Diana P. Rodriguez, ${ }^{2}$ \\ Catherine E. Cottrell, ${ }^{1,3,4}$ Jeffrey R. Leonard, ${ }^{4,5,6}$ Jonathan L. Finlay, ${ }^{4,7}$ \\ and Elaine R. Mardis ${ }^{1,4,5}$

\begin{abstract}
${ }^{1}$ The Steve and Cindy Rasmussen Institute for Genomic Medicine, Abigail Wexner Research Institute at Nationwide Children's Hospital, Columbus, Ohio 43215, USA; ${ }^{2}$ Department of Radiology, Nationwide Children's Hospital and The Ohio State University College of Medicine, Columbus, Ohio 43210, USA; ${ }^{3}$ Department of Pathology, ${ }^{4}$ Department of Pediatrics, ${ }^{5}$ Department of Neurosurgery, The Ohio State University College of Medicine, Columbus, Ohio 43210, USA; ${ }^{6}$ Department of Neurosurgery, ${ }^{7}$ Division of Hematology/Oncology/Bone Marrow Transplantation, Nationwide Children's Hospital, Columbus, Ohio 43205, USA
\end{abstract}

Corresponding author: Elaine.Mardis@ nationwidechildrens.org

(c) 2021 Miller et al. This article is distributed under the terms of the Creative Commons Attribution-NonCommercial License, which permits reuse and redistribution, except for commercial purposes, provided that the original author and source are credited.

Ontology terms: glioma; neoplasm of the central nervous system

Published by Cold Spring Harbor Laboratory Press

doi:10.1101/mcs.a006023
Abstract In this follow-up report, we present updated information regarding a previously reported pediatric patient with a World Health Organization grade I ganglioglioma harboring a BRAF p.T599dup mutation (Cold Spring Harb Mol Case Stud 4: a002618). This patient, based on our initial finding, is receiving combination targeted therapy with a selective BRAF inhibitor (dabrafenib) plus MEK inhibitor (trametinib). The combination therapy was started after the patient experienced progressive tumor growth and worsening neurological symptoms, including visual changes, headaches, and peripheral neuropathy, despite 9 months of treatment with adjuvant chemotherapy (vinblastine). The patient has been receiving dabrafenib plus trametinib for 15 months and continues to have stable disease as well as improved neurological symptoms. Although combinatorial therapy targeting BRAF and MEK using dabrafenib and trametinib, respectively, is indicated for tumors harboring a BRAF p.V600E/K mutation, our report demonstrates efficacy of this combination in a nonV600E BRAF-mutated tumor. The identification of BRAF alterations may assist clinicians in determining alternative targeted treatment strategies, especially considering the paucity of effective treatments for primary brain tumors and the poor prognosis associated with many central nervous system (CNS) diagnoses. Additional case studies or larger cohort reports will continue to clarify the efficacy of BRAF and/or MEK inhibitors in patients whose tumors harbor a BRAF alteration.

\section{INTRODUCTION}

BRAF alterations are commonly observed in many tumor types, the most frequent of which is BRAF p.Val600Glu (p.V600E). This variant is detected most frequently in melanomas and non-small-cell lung cancers and at a lower incidence in central nervous system (CNS) tumors (Davies et al. 2002; Schreck et al. 2019). Several other BRAF mutations, including p. Val600Lys (p.V600K) as well as other activating variants occurring within the kinase domain, have been identified as oncogenic drivers in different human cancers. Specifically, in pediatric low-grade gliomas, BRAF alterations are the most commonly identified molecular event, most of which are p.V600E or KIAA1549-BRAF gene fusions (Schindler et al. 2011; Ryall et al. 2017). 
COLD SPRING HARBOR Molecular Case Studies
Targeted therapy in ganglioglioma with BRAF T599dup
BRAF belongs to the RAF family of protein kinases and, when activated, phosphorylates downstream targets MEK1/2, which in turn phosphorylate and activate ERK1/2. This leads to activation of a variety of nuclear transcription factors and subsequent increased cell proliferation, survival, and tumor growth. A BRAF p.V600E/K variant results in constitutively activated kinase activity and increased downstream MEK/ERK activation (Wan et al. 2004). U.S. Federal Drug Administration (FDA)-approved therapies designed to inhibit BRAF (BRAFi) and other components in the RAS/MAPK pathway (MEKi) have demonstrated remarkable responses in phase II/III clinical trials in the treatment of many malignancies, including melanoma and thyroid cancer (Flaherty et al. 2012; Long et al. 2017; Subbiah et al. 2018). In particular, combination therapy with dabrafenib (BRAFi) and trametinib (MEKi) is FDA-approved for use in patients with BRAF p.V600E/K in melanoma, as well as BRAF p.V600E in non-small-cell lung cancer and thyroid cancer. This combinatorial therapy has even proven effective with off-label use in patients with BRAF p.V600E-mutated high-grade gliomas (Johanns et al. 2018; Schreck et al. 2018). The value of combination targeted therapy aimed at BRAF alterations other than p.V600E/K needs further study and more clinical evidence to determine efficacy of such drugs.

Previously, we described identification of a BRAF variant resulting in duplication of threonine at codon 599 [NM_004333.4:c.1794_1796dup (p.T599dup)] in a pediatric World Health Organization (WHO) grade I ganglioglioma (Miller et al. 2018) using genome sequencing. Prior in vitro studies of p.T599dup demonstrated kinase activity and cellular MEK/ERK activation potential comparable to that of p.V600E (Eisenhardt et al. 2011). In this follow-up report, we describe the patient's positive response to combination therapy with a selective BRAFi (dabrafenib) and MEKi (trametinib), which was initiated after she experienced progressive tumor growth and worsening neurological symptoms despite treatment with adjuvant chemotherapy (vinblastine).

\section{RESULTS}

An adolescent female initially presented with blurred vision, papilledema, and hydrocephalus and was diagnosed with a probable low-grade glioma based on magnetic resonance imaging (MRI). The patient was treated for hydrocephalus and followed with MRI surveillance every 3-4 mo, which showed gradual enlargement of the midbrain mass. Although the patient experienced no new neurological signs or symptoms, a partial resection was performed $4 \mathrm{yr}(48 \mathrm{mo})$ after diagnosis because of the progressive imaging changes. Pathological examination showed a low-grade brain tumor: ganglioglioma, WHO grade I. Subsequent genome sequencing of the tumor specimen revealed a BRAFp.T599dup mutation (Miller et al. 2018).

Routine MRI surveillance again showed enlargement of the tumor over the next 6 months following the partial resection (Table 1A). The radiologist reported increases in the size and degree of contrast enhancement of the midbrain mass (Fig. 1A,B). Vinblastine was used (6 $\mathrm{mg}$ per $\mathrm{m}^{2}$ of body surface area per dose) and administered intravenously every $2 \mathrm{wk}$. The patient was compliant with vinblastine treatments and continued MRI imaging every 3-4 mo after starting the chemotherapy regimen. The tumor was not responsive to vinblastine and demonstrated interval growth over time (Table 1B). In addition, the patient experienced worsening vision and debilitating peripheral neuropathy, for which she was prescribed gabapentin ( $900 \mathrm{mg}$ twice a day). After 9 mo of vinblastine, the radiologist noted increased size of solid and cystic components of the mass, and treatment with vinblastine was discontinued (Fig. 1C,D). The patient had a near-complete resection of the enhancing tissue in the central lesion (Fig. 1E,F). We obtained tumor-extracted DNA from this nearcomplete resection and performed deep, targeted sequencing of the p.T599dup BRAFvariant. The allele frequency of the BRAF variant detected in the more recent resection (5.9\%) 
Table 1. Interpretation of MRI findings related to type and time of treatment

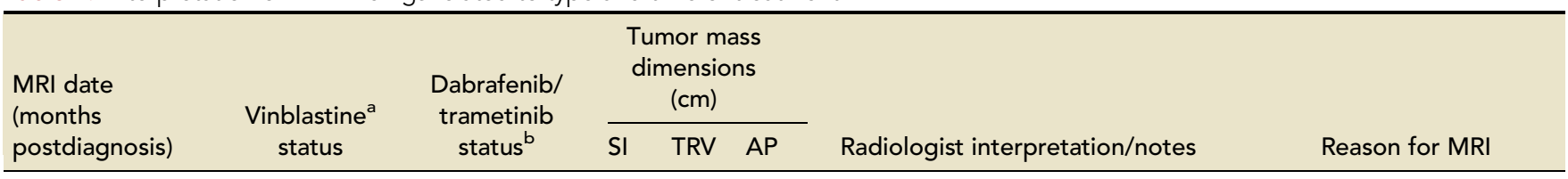

A

Initial diagnosis Prio

$47 \mathrm{mo}$

Prior

Prior

$50 \mathrm{mo}$

$52 \mathrm{mo}$

Prior

Prior

B

$57 \mathrm{mo}$

$60 \mathrm{mo}$

$63 \mathrm{mo}$

Prior

Prior

$6 \quad 2.7$

$1.8 \quad 2.5$

Prior

$$
\text { (3 mo) }
$$

Active

(6 mo)

Active

Prior

Prior

$1.7 \quad 2.6$
$1.7 \quad 1.5$

$2.0 \quad 2.5 \quad 2.6$

2.12
51.5
No significant contrast enhancement associated with this lesion, and no abnormal contrast enhancement appreciated in the remainder of the brain

2 prolongation mass lesion centered within the midbrain; increased in size since the previous study

Mass lesion minimally increased in size compared to previous imaging; central portion of the lesion shows increased T2 signal compared to the peripheral portion and enhances diffusely with contrast Mass unchanged to slightly decreased in size when compared to previous imaging

xpansile, T2 prolongation mass lesion with slight decrease in size; central area of the mass shows increased T2 signal and contrast enhancement

(9 mo)

Stopped

Prior

$65 \mathrm{mo}$

Stopped

Prior

NA NA

2.7

Complex appearing mass within the midbrain, with measurements comparable to prior imaging

$2.0 \quad 2.8 \quad 2.6 \quad$ Increased size of mass compared to most recent imaging.

$\begin{array}{llll}2.6 & 3.2 & 2.8 & \text { Increased size of both the solid and }\end{array}$ cystic components of the midbrain mass compared to previous MRI

$2.9 \quad 3.5 \quad 2.8 \quad$ Slight further increase in size of the cystic portion of the mixed solid and cystic mass; enhancing solid component appears unchanged

Almost complete resection of the enhancing tissue in the central lesion; residual crescent of $\mathrm{T} 2$ signal abnormality is still concerning for nonenhancing tumor

$68 \mathrm{mo} \quad$ Stopped Prior
New avidly enhancing nodules along the anterior aspect of the mass, suggestive of tumor progression.
Sudden onset nystagmus and loss of vision in left eye

Preoperative evaluation prior to initial biopsy and tumor resection

Routine surveillance

Evaluation prior to commencing chemotherapy

Routine surveillance

Disease assessment/ postchemotherapy commencement

Routine surveillance

Routine surveillance

Routine surveillance

Intraoperative during tumor resection

Routine surveillance

(Continued on next page.) 


\begin{tabular}{|c|c|c|c|c|c|c|c|}
\hline \multirow{2}{*}{$\begin{array}{l}\text { MRI date } \\
\text { (months } \\
\text { postdiagnosis) }\end{array}$} & \multirow{2}{*}{$\begin{array}{l}\text { Vinblastine } \\
\text { status }\end{array}$} & \multirow{2}{*}{$\begin{array}{l}\text { Dabrafenib/ } \\
\text { trametinib }^{\text {status }}{ }^{b}\end{array}$} & \multicolumn{3}{|c|}{$\begin{array}{l}\text { Tumor mass } \\
\text { dimensions } \\
\quad(\mathrm{cm})\end{array}$} & \multirow[b]{2}{*}{ Radiologist interpretation/notes } & \multirow[b]{2}{*}{ Reason for MRI } \\
\hline & & & SI & TRV & AP & & \\
\hline \multicolumn{8}{|l|}{ C } \\
\hline $71 \mathrm{mo}$ & Stopped & Active (2 mo) & 1.6 & 2.2 & 2.3 & $\begin{array}{l}\text { Similar in size with decreased } \\
\text { enhancement }\end{array}$ & Routine surveillance \\
\hline 74 mo & Stopped & Active (5 mo) & 1.5 & 2.2 & 2.2 & Stable appearance of residual tumor & Routine surveillance \\
\hline $78 \mathrm{mo}$ & Stopped & Active (9 mo) & 1.4 & 2.0 & 2.2 & No evidence of tumor progression & Routine surveillance \\
\hline $81 \mathrm{mo}$ & Stopped & Active (12 mo) & 1.4 & 2.0 & 2.2 & $\begin{array}{l}\text { Essentially unchanged in imaging } \\
\text { appearance and tumor dimensions }\end{array}$ & Routine surveillance \\
\hline 84 mo & Stopped & Active (15 mo) & 1.4 & 2.0 & 2.0 & $\begin{array}{l}\text { Stable nonenhancing, lobulated } \\
\text { midbrain mass; unchanged mild to } \\
\text { moderate enlargement of the } \\
\text { lateral and third ventricles }\end{array}$ & Routine surveillance \\
\hline
\end{tabular}

"Prior" refers to the time period before therapy initiation.

(MRI) Magnetic resonance imaging, (SI) superior to inferior, (TRV) transverse, (AP) anteroposterior.

a Vinblastine dosage, $6 \mathrm{mg} / \mathrm{m}^{2} /$ dose intravenously every $2 \mathrm{wk}$.

${ }^{b}$ Dabrafenib dosage, $150 \mathrm{mg}$ twice a day; trametinib dosage, $1.5 \mathrm{mg}$ twice a day.

was retained at a slightly higher, but similar, frequency as detected in the patient's initial tumor resection (5.2\%).

Follow-up imaging 3 mo postresection showed new contrast enhancement and evidence of tumor growth (Fig. 1G,H). The patient was switched to combination targeted therapy with BRAFi dabrafenib (150 mg twice a day) and MEKi trametinib (1.5 mg twice a day), nearly 6 yr (69 mo) after her initial diagnosis. Two months following commencement of the combination targeted therapy, the mass showed a decrease in size and contrast enhancement (Fig. 1I,J). Routine MRIs continue to show diminished enhancing portions and decreased measurements of the tumor dimensions (Table 1C). One year after starting BRAFi/MEKi, ophthalmologic exams demonstrated overall improvement in the patient's visual fields, and she has also experienced resolution of peripheral sensory neuropathy and was able to discontinue use of gabapentin. The patient has tolerated dabrafenib and trametinib well, except for development of painful nodules under the skin of her thighs. She occasionally stops treatment because of the skin toxicity and resumes after resolution of the nodules, usually within a 2-wk time period. The patient has been on the new combination therapy for $15 \mathrm{mo}$ and continues to have stable disease with sustained tumor shrinkage and no new enhancing portions within the mass (Fig. 1K,L).

\section{DISCUSSION}

Our report demonstrates the sensitivity of a non-V600E BRAF mutation in a low-grade glioma to treatment with combination targeted therapy (BRAFi/MEKi). We identified two other reports in which patients (one with melanoma and one with lung cancer) each harboring the BRAF p.T599dup received dabrafenib/trametinib and demonstrated a positive clinical response including stable disease in the lung cancer case and partial remission in the melanoma case (Marchand et al. 2018; Mu et al. 2020). Furthermore, other studies have reported successful outcomes of dabrafenib/trametinib in pediatric patients with BRAF p.V600E low-grade gliomas, demonstrating safe and effective use in treating brain tumors (Marks et al. 2018; Hargrave et al. 2019). 

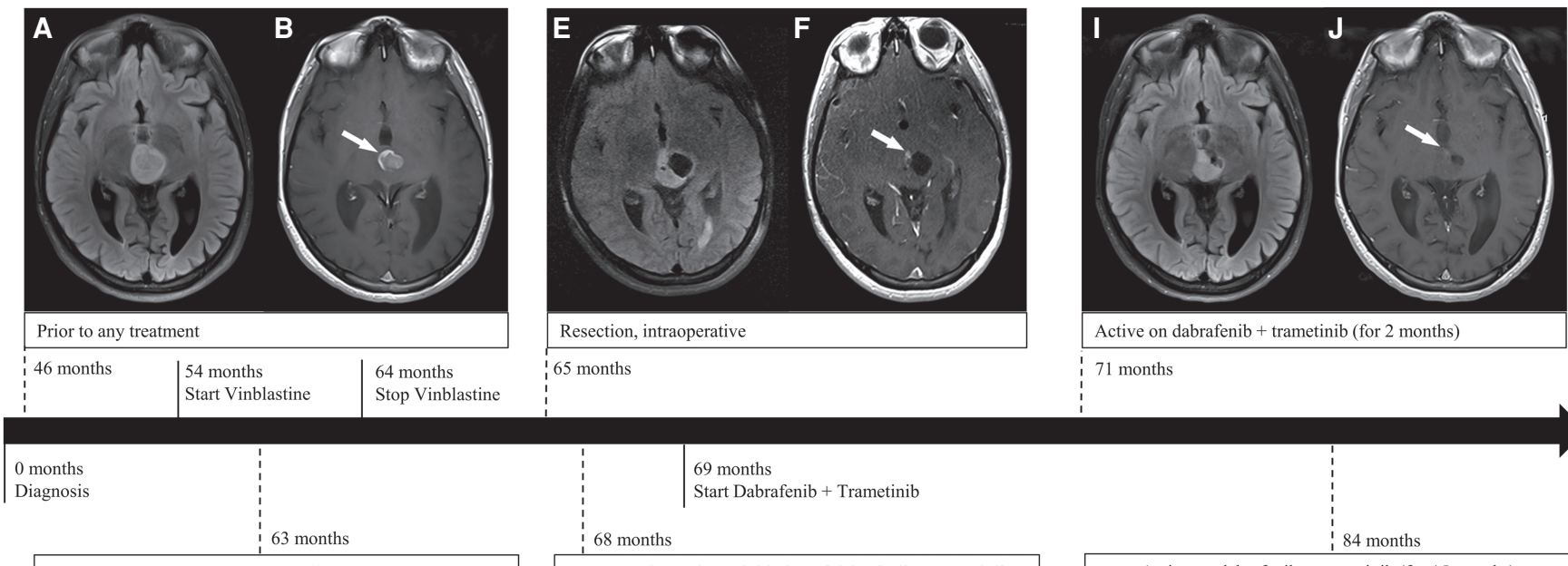

Active on dabrafenib + trametinib (for 2 months) 71 months
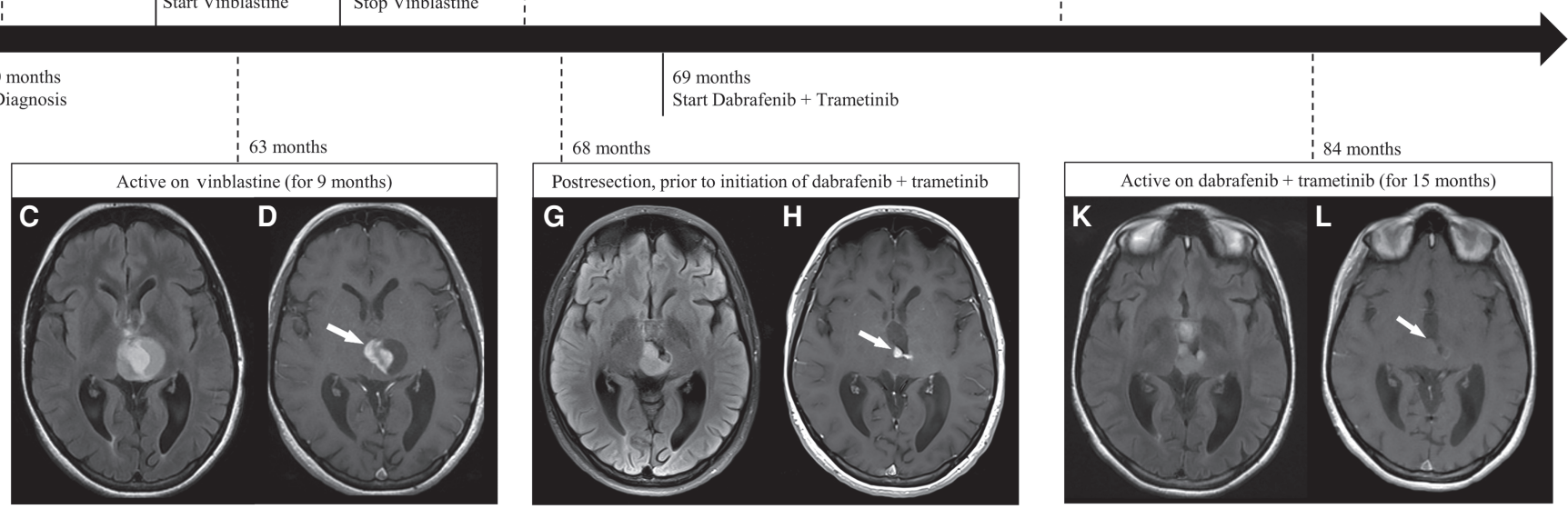

Figure 1. Radiographic features of the tumor related to type and time of treatment. Prior to vinblastine and dabrafenib/trametinib through 46 mo postdiagnosis: Axial T2-FLAIR image (A) shows a well-circumscribed T2-hyperintense midbrain mass, reported as increased in size; postcontrast axial T1-W image (B) shows intense peripheral rim enhancement (arrow) and central diffuse enhancement. Active on vinblastine (for $9 \mathrm{mo}$ ) and prior to dabrafenib/trametinib through 63 mo postdiagnosis: Axial T2-FLAIR image (C) shows increased size of solid and cystic components of the mass; postcontrast axial T1-W image (D) shows increased central area of enhancement (arrow). Intraoperative, postresection, 65 mo postdiagnosis: Axial T2-FLAIR image $(E)$ shows partial resection of solid and cystic components of the mass; postcontrast axial T1-W image (F) shows a residual sub centimeter enhancing focus (arrow). Three months postresection and prior to initiation of dabrafenib/trametinib through 68 mo postdiagnosis: Axial T2-FLAIR image $(G)$ shows increased size of the anterior solid component of the mass; postcontrast axial T1-W image $(H)$ shows two new nodular enhancing foci (arrow). Stopped vinblastine and active on dabrafenib/trametinib (for $2 \mathrm{mo}$ ) through 71 mo postdiagnosis: Axial T2-FLAIR image $(I)$ shows decreased size of the mass; postcontrast axial T1-W image $(J)$ shows significantly decreased enhancement (arrow). Stopped vinblastine and active on dabrafenib/trametinib (for $15 \mathrm{mo}$ ) through 84 mo postdiagnosis: Axial T2-FLAIR image $(K)$ shows further decrease in size of the mass; postcontrast axial T1-W image $(L)$ shows minimal to no enhancement (arrow).

Identification of different treatment paradigms for pediatric brain tumors is critical, as these tumor types remain the leading cause of disease-related death in children (Curtin et al. 2016). MEKi are attractive because BRAF alterations resulting in downstream activation of the RAS/MAPK pathway are present in a significant subset of pediatric brain tumors (pilocytic astrocytomas, 10\%-15\%; pleomorphic xanthoastrocytomas, 50\%-60\%; gangliogliomas, 20\%-60\%) and offer a useful target for therapy (Schindler et al. 2011; Pekmezci et al. 2018). There is a growing use and appreciation for efforts to molecularly characterize malignant and progressive tumors, with the intent to identify targetable alterations for each patient. A recent investigation of publicly available databases revealed 20 different known activating BRAF mutations reported in pediatric gliomas (Schreck et al. 2019). In our patient, identification of the mutation coupled with published functional knowledge derived from in vitro data were critical for determining the potential benefit of targeted therapy. Additional 
COLD SPRING HARBOR Molecular Case Studies
Targeted therapy in ganglioglioma with BRAF T599dup

case studies or larger cohort reports will continue to clarify the efficacy of BRAFi and/or MEKi in patients whose tumors harbor a BRAF alteration.

\section{METHODS}

\section{MRI}

All brain MRI scans were performed without and with intravenous administration of a gadolinium-based contrast agent, Gadobutrol (Gadavist). Imaging was obtained on the following three Tesla scanners using a 32-channel head coil: Siemens Magnetom Skyra and Siemens PRISMA (Siemens Healthcare), and General Electric Discovery MR750 (General Electric Healthcare). The following 1.5-Tesla scanners were used as well, using a 12-channel head coil: General Electric Signa Explorer and General Electric Signa HDxt (General Electric Healthcare). Images were interpreted by a board-certified pediatric neuroradiologist (D.P.R.). MRI factors were incorporated as factors for assessing the patient's response to treatment in accordance with the Response Assessment in Neuro-Oncology (RANO) criteria (Wen et al. 2017).

\section{Targeted Sequencing}

Genomic DNA (10 ng) was used as a template for polymerase chain reaction (PCR) designed to amplify a 300-bp product that includes the BRAF p.599 position. BRAF exon 15 primers: forward (5'-ACTCTTCATAATGCTTGCTCTGA-3') and reverse (5'-AGTAACTCAGC AGCATCTCAGG-3'). PCR was performed using 2× Q5 MM (New England BioLabs) and $200 \mathrm{nM}$ primers with the following conditions: $30^{\prime \prime}$ at $98^{\circ} \mathrm{C}, 30$ cycles of $10^{\prime \prime}$ at $98^{\circ} \mathrm{C}, 30^{\prime \prime}$ at $60^{\circ} \mathrm{C}, 30^{\prime \prime}$ at $72^{\circ} \mathrm{C}$, and a final extension of $5^{\prime}$ at $72^{\circ} \mathrm{C}$. Amplified products were purified using 1.8 $\times$ SPRIselect, followed by end repair and dA-tailing using NEBNext Ultra II DNA Library Prep kit reagents. The reaction was followed by adapter ligation with unique molecular identifier (UMI)-IDT-indexed adaptors (Integrated DNA Technologies). Adaptor-ligated samples were purified using 1.2× SPRIselect and used for library amplification with Q5 MM and Illumina P5/P7 primer mix. A post-PCR 1.2× SPRlselect cleanup was performed, and libraries were pooled and sequenced on Illumina iSeq100 to achiever average read depth of $200,000 \times$ at the BRAF variant site.

\section{ADDITIONAL INFORMATION}

\section{Ethics Statement}

The patient was consented onto a translational research sequencing protocol, which was previously approved by the Institutional Review Board of The Research Institute at Nationwide Children's Hospital.

\section{Acknowledgments}

The authors thank the patient for participation in our study. We acknowledge Brad Hoehne, Radiology Digital Imaging Specialist, for preparation of figures. We acknowledge support from the Nationwide Foundation Innovation Fund.

Competing Interest Statement

The authors have declared no competing interest.

Received November 21, 2020; accepted in revised form February 22, 2021.

\section{Author Contributions}

J.R.L. and J.L.F. were responsible for patient clinical care. D.P.R. analyzed and interpreted patient MRI scans. K.E.M. and K.M.S. analyzed and interpreted patient clinical data. K.E.M., O.G., C.E.C., and E.R.M. drafted and edited the initial manuscript. All authors read and approved the case report as written. 


\section{REFERENCES}

Curtin S, Minino A, Anderson R. 2016. Declines in cancer death rates among children and adolescents in the United States, 1999-2014. NCHS data brief no. 257. National Center for Health Statistics, Hyattsville, MD.

Davies H, Bignell GR, Cox C, Stephens P, Edkins S, Clegg S, Teague J, Woffendin H, Garnett MJ, Bottomley W, et al. 2002. Mutations of the BRAF gene in human cancer. Nature 417:949-954. doi:10.1038/nature00766

Eisenhardt AE, Olbrich H, Roring M, Janzarik W, Anh TN, Cin H, Remke M, Witt H, Korshunov A, Pfister SM et al. 2011. Functional characterization of a BRAF insertion mutant associated with pilocytic astrocytoma. Int J Cancer 129: 2297-2303. doi:10.1002/ijc.25893

Flaherty KT, Robert C, Hersey P, Nathan P, Garbe C, Milhem M, Demidov LV, Hassel JC, Rutkowski P, Mohr P, et al. 2012. Improved survival with MEK inhibition in BRAF-mutated melanoma. N Engl J Med 367: 107114. doi:10.1056/NEJMoa1203421

Hargrave DR, Bouffet E, Tabori U, Broniscer A, Cohen KJ, Hansford JR, Geoerger B, Hingorani P, Dunkel IJ, Russo MW, et al. 2019. Efficacy and safety of dabrafenib in pediatric patients with BRAF V600 mutationpositive relapsed or refractory low-grade glioma: results from a phase I/Ila study. Clin Cancer Res 25: 7303-7311. doi:10.1158/1078-0432.CCR-19-2177

Johanns TM, Ferguson CJ, Grierson PM, Dahiya S, Ansstas G. 2018. Rapid clinical and radiographic response with combined dabrafenib and trametinib in adults with BRAF-mutated high-grade glioma. J Natl Compr Canc Netw 16: 4-10. doi:10.6004/jnccn.2017.7032

Long GV, Hauschild A, Santinami M, Atkinson V, Mandala M, Chiarion-Sileni V, Larkin J, Nyakas M, Dutriaux C, Haydon A, et al. 2017. Adjuvant dabrafenib plus trametinib in stage III BRAF-mutated melanoma. N Engl J Med 377: 1813-1823. doi:10.1056/NEJMoa1708539

Marchand A, Tallet A, Collin C, Cormier B, Venel Y, Miquelestorena-Standley E, Machet L. 2018. A rare BRAF T599dup mutation conferring sensitivity to BRAF inhibitor in a patient with metastatic melanoma. $\mathrm{Br} J$ Dermatol 179: 528-529. doi:10.1111/bjd.16499

Marks AM, Bindra RS, DiLuna ML, Huttner A, Jairam V, Kahle KT, Kieran MW. 2018. Response to the BRAF/ MEK inhibitors dabrafenib/trametinib in an adolescent with a BRAF V600E mutated anaplastic ganglioglioma intolerant to vemurafenib. Pediatr Blood Cancer 65: e26969. doi:10.1002/pbc.26969

Miller KE, Kelly B, Fitch J, Ross N, Avenarius MR, Varga E, Koboldt DC, Boue DR, Magrini V, Coven SL, et al. 2018. Genome sequencing identifies somatic BRAF duplication c.1794_1796dupTAC;p.Thr599dup in pediatric patient with low-grade ganglioglioma. Cold Spring Harb Mol Case Stud 4: a002618. doi:10.1101/ mcs.a002618

Mu Y, Yang K, Hao X, Wang Y, Wang L, Liu Y, Lin L, Li J, Xing P. 2020. Clinical characteristics and treatment outcomes of 65 patients with BRAF-mutated non-small cell lung cancer. Front Oncol 10: 603. doi:10 .3389/fonc. 2020.00603

Pekmezci M, Villanueva-Meyer JE, Goode B, Van Ziffle J, Onodera C, Grenert JP, Bastian BC, Chamyan G, Maher OM, Khatib Z, et al. 2018. The genetic landscape of ganglioglioma. Acta Neuropathol Commun 6: 47. doi:10.1186/s40478-018-0551-z

Ryall S, Arnoldo A, Krishnatry R, Mistry M, Khor K, Sheth J, Ling C, Leung S, Zapotocky M, Guerreiro Stucklin A, et al. 2017. Multiplex detection of pediatric low-grade glioma signature fusion transcripts and duplications using the nanostring ncounter system. J Neuropathol Exp Neurol 76: 562-570. doi:10.1093/jnen/nlx042

Schindler G, Capper D, Meyer J, Janzarik W, Omran H, Herold-Mende C, Schmieder K, Wesseling P, Mawrin C, Hasselblatt $M$, et al. 2011. Analysis of BRAF V600E mutation in 1,320 nervous system tumors reveals high mutation frequencies in pleomorphic xanthoastrocytoma, ganglioglioma and extra-cerebellar pilocytic astrocytoma. Acta Neuropathol 121: 397-405. doi:10.1007/s00401-011-0802-6

Schreck KC, Guajardo A, Lin DDM, Eberhart CG, Grossman SA. 2018. Concurrent BRAF/MEK inhibitors in BRAF V600-mutant high-grade primary brain tumors. J Natl Compr Canc Netw 16: 343-347. doi:10 $.6004 /$ jnccn.2017.7052

Schreck KC, Grossman SA, Pratilas CA. 2019. BRAF mutations and the utility of RAF and MEK inhibitors in primary brain tumors. Cancers (Basel) 11: 1262. doi:10.3390/cancers11091262

Subbiah V, Kreitman RJ, Wainberg ZA, Cho JY, Schellens JHM, Soria JC, Wen PY, Zielinski C, Cabanillas ME, Urbanowitz G, et al. 2018. Dabrafenib and trametinib treatment in patients with locally advanced or metastatic BRAF V600-mutant anaplastic thyroid cancer. J Clin Oncol 36: 7-13. doi:10.1200/JCO.2017.73 .6785

Wan PT, Garnett MJ, Roe SM, Lee S, Niculescu-Duvaz D, Good VM, Jones CM, Marshall CJ, Springer CJ, Barford D, et al. 2004. Mechanism of activation of the RAF-ERK signaling pathway by oncogenic mutations of B-RAF. Cell 116: 855-867. doi:10.1016/S0092-8674(04)00215-6

Wen PY, Chang SM, Van den Bent MJ, Vogelbaum MA, Macdonald DR, Lee EQ. 2017. Response assessment in neuro-oncology clinical trials. J Clin Oncol 35: 2439-2449. doi:10.1200/JCO.2017.72.7511 


\section{COLD SPRING HARBOR Molecular Case Studies}

\section{Clinical response to dabrafenib plus trametinib in a pediatric ganglioglioma with BRAF p.T599dup mutation}

Katherine E. Miller, Kathleen M. Schieffer, Olivia Grischow, et al.

Cold Spring Harb Mol Case Stud 2021, 7: a006023 originally published online February 26, 2021 Access the most recent version at doi: $10.1101 / \mathrm{mcs} . a 006023$

References This article cites 18 articles, 4 of which can be accessed free at: http://molecularcasestudies.cshlp.org/content/7/2/a006023.full.html\#ref-list-1

License This article is distributed under the terms of the Creative Commons Attribution-NonCommercial License, which permits reuse and redistribution, except for commercial purposes, provided that the original author and source are credited.

Email Alerting Receive free email alerts when new articles cite this article - sign up in the box at the Service top right corner of the article or click here. 